\title{
Myoma morcellation and leiomyosarcoma panic
}

\author{
Vasilios Tanos • Hans Brölmann • Rudi Leon DeWilde • \\ Peter O'Donovan • Rudi Campo
}

Received: 2 September 2014 / Accepted: 17 December 2014 /Published online: 16 January 2015

(C) Springer-Verlag Berlin Heidelberg 2015

In a press release in April 2014, the US Food and Drug Administration [1] discourages doctors from using laparoscopic power morcellators in removing fibroids or myomatotic uteri in order to reduce the risk of sarcomatous cell escape. The USbased company Johnson \& Johnson, the largest manufacturer of the devices, halted global sales and distribution of power morcellators, while many hospitals in USA ban the use of morcellation under these circumstances. Patients' safety and wellbeing especially after surgery is a common and primary concern of surgeons and hospitals; however, there is no reliable

V. Tanos $\cdot$ H. Brölmann $\cdot$ R. L. DeWilde $\cdot$ P. O’Donovan $\cdot$ R. Campo Head IVF and Reproductive Surgery, Aretaeio Hospital, Nicosia, Cyprus

\section{Tanos $(\bowtie)$}

St. Georges Medical School, Nicosia University, Nicosia, Cyprus e-mail: v.tanos@aretaeio.com

H. Brölmann

Department of Obstetrics and Gynecology, VU University Medical Center, De Boelelaan 1117, 1181HV, Amsterdam, The Netherlands

\section{R. L. DeWilde}

Clinic of Gynecology, Obstetrics and Gynecological Oncology, University Hospital for Gynecology, Pius-Hospital Oldenburg Medical Campus University of Oldenburg, Oldenburg, Germany

\section{P. O’Donovan}

Obstetrics and Gynecological Oncology Yorkshire Clinic,

Bradford Road, Bingley BD16 1TW, West Yorkshire, UK

R. Campo

Past President European Society Gynaecological Endoscopy, European Academy for Gynecological Surgery, Diestsevest 43/0001, 3000 Leuven, Belgium way to determine if a uterine fibroid contains a sarcoma prior to removal. Patients should know that the use of laparoscopic power morcellation for hysterectomy or myomectomy may deteriorate their survival rate in case of a sarcoma, and they should discuss the risks and benefits of the available treatment options with their health care professionals.

The incidence of uterine sarcomas is extremely low, $0.23 \%$ [2], or according to FDA analysis of currently available data (1:350), $0.29 \%$. They are classified according to histological subtypes in order of decreasing incidence: leiomyosarcomas, endometrial stromal sarcomas (ESS) and 'other' sarcomas. The ESS are lesions within the endometrial cavity and diagnosis can be established prior to surgery by endometrial biopsy [3].

The rarity of these tumours has prevented the performance of large epidemiological studies to identify risk factors. Assuming an incidence of uterine sarcomas of $0.23 \%$ by applying the 'inverse rule of 3', a surgeon will need to perform 1304 laparoscopic interventions to observe at least one case of uterine sarcoma with $95 \%$ confidence. Imaging characteristics, tumour markers and other parameters indicating the risk of sarcoma are not available or not specific. In addition, the lack of uniform histologic criteria for diagnosing uterine leiomyosarcoma (ULMS) makes interpretation of the older studies difficult [4]. As the Stanford study [5] was the first to appreciate that the type of necrosis in a uterine smooth muscle tumour was of crucial importance, studies that preceded it did not evaluate the presence or absence of tumour cell necrosis. A leiomyosarcoma usually exhibits diffuse moderate-tosevere atypia, a mitotic count of $>10 \mathrm{MFs} / 10 \mathrm{HPFs}$ and tumour cell necrosis. A tumour with any two of these features 
is clinically malignant often enough to warrant a diagnosis of leiomyosarcoma [6].

The majority of leiomyosarcomas are considered to arise de novo [7], most frequently located in uterine myometrium arising from the connective tissue of uterine blood vessels or in a preexisting leiomyoma with aggressive clinical course and poor prognosis. The spread of leiomyosarcomatous cells are primarily to the myometrium, pelvic blood vessels and lymphatics, adjacent pelvic structures, abdomen and distantly, most often in the lungs [8]. The ESS there is a higher relapse rate, though due to indolent behaviour, no impact on mortality. Patients need lifelong treatment and some are likely to succumb after many years. Morcellation of undifferentiated sarcoma and endometrial stromal sarcoma is also of importance.

Data regarding parity, age of menarche and menopause as risk factors are inconclusive. ULMS occur primarily in women 40 to 60 years of age. Among 1332 patients operated for uterine myoma with mean age of 43 years, only one woman at age of 30 was diagnosed with sarcoma, two women at age 30 and 50 had an endometrial stromal sarcoma while nine at ages 64-86 years were also found to have sarcomas [2]. There is approximately a two- to three-fold higher incidence of ULMS among African-American women compared to Caucasian women [9-11], and a history of pelvic irradiation is noted in $5-10 \%$ of patients [12]. According to Parker WH et al. in 1994 [2], who reviewed 26 studies, in 580 patients with suspected uterine sarcomas, the most common symptom was abnormal uterine bleeding, followed by pain and the presence of a pelvic mass. On pelvic examination, the uterus is often enlarged, and in some cases, part of the tumour may prolapse through the cervical os into the vaginal canal.

Seidman MA et al. in 2012 [13] reported 1091 cases of uterine morcellation performed from 2005 to 2010 . The rate of unexpected sarcoma after the laparoscopic morcellation procedure was $0.09 \%$, nine-fold higher than the rate currently quoted in the USA to patients during pre-procedure briefing. Follow-up laparoscopies revealed disseminated disease in $64.3 \%$ of all tumours, $4 / 4$ smooth muscle tumour of uncertain malignant potential (STUMP), 4/7 ULMS, 0/1 endometrial stromal sarcoma (ESS), 1/1 cellular leiomyoma (CL) and 0/6 atypical leiomyomas (AL). Only disseminated leiomyosarcoma were associated with mortality. The authors concluded that uterine morcellation carries a risk of disseminating unexpected malignancy associated with increased mortality much higher than appreciated currently.

The current treatment of myomas varies and is often adjusted to the wish of the patient. Most of the times, the opinion of the patient is highly respected since women would like to preserve their uterus, although doctor's advice might be hysterectomy. Most gynaecologists propose hysterectomy if the patient is not interested in conserving fertility to be pregnant as the best option due to future frequent development of new myomas but also in rare cases due to suspected ULMS. The perimenopausal patients without or with limited symptoms are usually managed expectantly since upon entering menopause, myomas reduce in size. Women with infertility, metrorrhagia and/or pelvic pain undergo hysteroscopic or laparoscopic myomectomy and others, laparoscopic total or subtotal hysterectomy. Other treatment modalities used like thermal ablation of fibroids or endometrium by vaginal or abdominal route, uterine artery embolization and medical approaches such as GnRHa and ullipristal acetate cannot be abandoned just because of fear of LMS. In cases of huge uterus inoperable by laparoscopy and in cases suspected for sarcoma, hysterectomy should be performed by laparotomy.

Unexpected diagnoses of leiomyoma variants or atypical and malignant smooth muscle tumours occurred in $1.2 \%$ of cases using power morcellation for uterine masses clinically presumed to be 'fibroids' [13]. Gynaecologists should follow the protocol for postmenopausal or irregular bleedings and perform endometrial biopsies prior to any further surgery in order to rule out endometrial malignancy. Parameters such as the size of myomas $>10 \mathrm{~cm}$ in diameter, age of the patients around 50, imaging evidence of necrosis and neoangiogenesis and serological markers such as LDH could isolate patients at high risk for LMS and could probably be advised to have TAH. MRI characteristics were analysed in 12 patients with benign leiomyomas but initially, clinically suspected as LMS and compared to nine women with pathologically proven LMS and three with STUMP. The size, location, signal intensity and contrast enhancement of the tumours were studied on an individual basis. The authors concluded that more than 50 $\%$ of high signal on T2-weighted images and the presence of any small high-signal areas on T1-weighted images with unenhanced pockets were considered MRI suggestive for STUMPS and LMS [14].

Peri- and postmenopausal symptomatic women with myomas insisting on undergoing myomectomy on demand or supracervical hysterectomy should be informed and consented prior to operation. It is important that morcellation devices should not be applied to remove suspected malignant tissues. Low-risk patients should be adequately informed preoperatively that morcellation can spread cancer cells in the unlikely case of hidden malignancy and not be falsely assured that uterine masses are not cancerous. In an objective way, the doctor should explain that at this moment, there is no way to completely exclude cancerous cells within myometrium or in a myoma. Conservative myomectomy of a LMS by laparotomy would probably also worsened patients' survival compared to direct hysterectomy. The efficacy and safety of morcellation within a laparoscopic bag falls within those new techniques which have been developed by the process of 'trial and error'. Unfortunately there is no extensive, prospective and reliable research data to support its application in the daily surgery practice. Patients with fibroids should be individually assessed and treated according to currently available data regarding ULMS. 
Inadequate and/or exaggerated counselling towards hysterectomy or abdominal myomectomy for all myomas and large uterus referring to sarcoma risk seems wrong and unethical. Simply to cover our medical legal aspects, we expose the patient to psychological distress and unnecessary worries and overtreat them. It is unfair and unjustified to operate on a young patient with a small myoma potentially causing infertility by laparotomy, raising the risk of postoperative adhesions, discomfort and longer hospitalization stay. Increased awareness of sarcoma risk is essential, but objective and correct reasoning should prevail on any decision prior to extent and type of surgery. Women with myomas suspicious of LMS should be properly informed about the options for myomectomy versus hysterectomy and laparoscopic surgery versus laparotomy.

Patient triage based on strict criteria of knowledge and practical experience is the only way to minimize misdiagnosis and secure patients' safety. The leiomyoma growing in postmenopausal women; single leiomyomas of rapid significant growth, with necrosis reported in MRI; increased vascularisation and not responding to GnRH analogues or embolization; and increased serum LDH isoenzyme are all suspicious for sarcomatous changes. At this moment, we are not able to prevent morcellation of all sarcomas. However, appropriate triage will definitely reduce the numbers of inappropriate morcellation.

\section{ESGE actions}

The European Society of Gynaecological Endoscopy (ESGE), in an effort to be objective as possible, initiated a survey among its members to collect data from each centre and surgeon separately. The first part of the survey is looking into the frequency of morcellated malignancies and safety issues using a morcellator. The second part of the survey will try to identify the conditions and characteristics of these rare malignant cases that happened to be morcellated. A survey for morcellator adverse events is also included.

A committee has been established with experienced surgeons and researchers well known for their interest and surgical abilities in myomectomies and hysterectomies in order to bring evidence-based data, exchange ideas and prepare scientific material to form a pre- and intraoperative protocol of myoma and myomatotic uterus follow-up and best way of treatment. The major task is to analyse data and review the literature in order to report if possible the real perspectives of ULMS frequency and course of the disease and suggest a preoperative workup in cases with myomas and large uterus. Based on retrieved data and experts' opinion, the ESGE will formulate recommendations and promote its implementation. A consensus meeting on sarcoma treatment will then be organized and all gynaecologists interested are invited to participate.

Conflict of interest Dr Campo Rudi is a consultant for Karl Storz endoscope. Prof. Rudi Leon De Wilde receives reimbursement of travel expenses to international congresses by the Karl Storz Company. Prof. Hans Brölmann does research project with Olympus, Gynesonics and Gedeon-Richter without any personal fees. Prof. Peter O'Donovan provides consultancy advice to both Karl Storz and Lina Medical in the last year in the field of ambulatory gynaecology nothing linked with morcellation. Prof. Vasilios Tanos declares no conflict of interest.

The authors declare that their relation with the companies mentioned above have no impact upon the scientific value and the content of the submitted article entitled 'Myoma Morcellation and Sarcoma Panic' assigned by manuscript number GYSU-D-14-00045.

\section{References}

1. FDA Safety Communication: Laparoscopic Uterine Morcellation, April 2014.888-INFO-FDA, dsmica@fda.hhs.gov Nat Institutes of Health: Uterine Fibroids Fact Sheet, March 2013.

2. Parker WH, Fu YS, Berek JS (1994) Uterine sarcoma in patients operated on for presumed leiomyoma and rapidly growing leiomyoma. Obstet Gynecol 83:414

3. Lurain JR, Piver MS (1992) Uterine sarcomas: clinical features and management. In: Coppleson John M, Paul M, Martin T (eds) Gynecologic oncology. Chruchill Livingstone, London, pp 827-840

4. Van Dinh T, Woodruff JO (1982) Leiomyosarcoma of the uterus. Am J Obstet Gynecol 144:817

5. Bell SW, Kempson RL, Hendrickson MR (1994) Problematic uterine smooth muscle neoplasms. A clinicopathologic study of 213 cases. Am J Surg Pathol 18:535-558

6. Ip PPC, Tse KY, Tam KF (2010) Uterine smooth muscle tumors other than the ordinary leiomyomas and leiomyosarcomas: a review of selected variants with emphasis on recent advances and unusual morphology that may cause concern for malignancy. Adv Anat Pathol 17(2):93-112

7. Serur E, Lakhi N (2011) Laparoscopic hysterectomy with manual morcellation of the uterus an original technique that permits the safe and quick removal of a large uterus. Am J Obstet Gynecol 204:566. doi:10.1016/j.ajog.2011.03.042, e1-2

8. Reichardt P (2012) The treatment of uterine sarcomas. Ann Oncol 23(10):x151-7

9. Harlow BL, Weis NS, Lofton S (1986) The epidemiology of sarcoma of the uterus. J Natl Cancer Inst 76:399

10. Brooks SE, Zhan M, Cote T, Baquet CR (2004) Surveillance, epidemiology, and end results analysis of 2677 cases of uterine sarcoma 1989-1999. Gynecol Oncol 93:204

11. Arrastia CD, Fruchter RG, Clark M et al (1997) Uterine carcinosarcomas: incidence and trends in management and survival. Gynecol Oncol 65:158

12. Meredith RF, Eisert DR, Kaka Z et al (1986) An excess of uterine sarcoma after pelvic irradiation. Cancer 58:2003

13. Seidman MA, Oduyebo T, Muto MG, Crum CP, Nucci MR et al (2012) Peritoneal dissemination complicating morcellation of uterine mesenchymal neoplasms. PLoS ONE 7(11):e50058. doi:10.1371/ journal.pone. 0050058

14. Tanaka YO, Nishida M, Tsunoda H, Okamoto Y, Toshikawa H (2004) Smooth muscle tumors of uncertain malignant potential and leiomyosarcomas of the uterus: MR findings. J Magnetic Resonance Imaging 20(6):998-1007 